\title{
Fractional Killing-Yano Tensors and Killing Vectors Using the Caputo Derivative in Some One- and Two-Dimensional Curved Space
}

\author{
Ehab Malkawi ${ }^{1}$ and D. Baleanu ${ }^{2,3,4}$ \\ ${ }^{1}$ Department of Physics, United Arab Emirates University, 15551 Al Ain, UAE \\ ${ }^{2}$ Department of Chemical and Materials Engineering, Faculty of Engineering, King Abdulaziz University, P.O. Box 80204, \\ Jeddah 21589, Saudi Arabia \\ ${ }^{3}$ Department of Mathematics and Computer Sciences, Faculty of Arts and Sciences, Cankaya University, 06530, Balgat, Ankara, Turkey \\ ${ }^{4}$ Institute of Space Sciences, Magurele 76900, Bucharest, Romania \\ Correspondence should be addressed to Ehab Malkawi; emalkawi@uaeu.ac.ae
}

Received 30 January 2014; Accepted 25 February 2014; Published 24 March 2014

Academic Editor: Xiao-Jun Yang

Copyright (C) 2014 E. Malkawi and D. Baleanu. This is an open access article distributed under the Creative Commons Attribution License, which permits unrestricted use, distribution, and reproduction in any medium, provided the original work is properly cited.

The classical free Lagrangian admitting a constant of motion, in one- and two-dimensional space, is generalized using the Caputo derivative of fractional calculus. The corresponding metric is obtained and the fractional Christoffel symbols, Killing vectors, and Killing-Yano tensors are derived. Some exact solutions of these quantities are reported.

\section{Introduction}

The tool of the fractional calculus started to be successfully applied in many fields of science and engineering (see, e.g., [112] and the references therein). Fractals and its connection to local fractional vector calculus represents another interesting field of application (see, e.g., $[13,14]$ and the references therein). Several definitions of the fractional differentiation and integration exist in the literature. The most commonly used are the Riemann-Liouville and the Caputo derivatives. The Riemann-Liouville derivative of a constant is not zero while Caputo's derivative of a constant is zero. This property makes the Caputo definition more suitable in all problems involving the fractional differential geometry $[15,16]$. The Caputo differential operator of fractional calculus is defined as $[1-8]$

$$
{ }_{a} D_{x}^{\alpha} f(x) \equiv \begin{cases}\frac{1}{\Gamma(n-\alpha)} & \\ \times \int_{a}^{x}(x-u)^{n-\alpha-1} \frac{d^{n} f(u)}{d u^{n}} d u, & n-1<\alpha<n \\ \frac{d^{n}}{d x^{n}} f(x), & \alpha=n,\end{cases}
$$

where $\Gamma(\cdot)$ is the Gamma function and $x>a$. In this work, we consider the case $a=0, n-1<\alpha \leq n$. For the power function $x^{p}, p \in R$, the Caputo fractional derivative satisfies

$$
D_{x}^{\alpha} x^{p}= \begin{cases}\frac{\Gamma(p+1)}{\Gamma(p-\alpha+1)} x^{p-\alpha} & \\ 0, & p=0,1,2, \ldots, n-1 .\end{cases}
$$

The role played by Killing and Killing-Yano tensors for the geodesic motion of the particle and the superparticle in a curved background was a topic subjected to an intense debate during the last decades [17-26]. In [27] a generalization of exterior calculus was presented. Besides, the quadratic Lagrangians are introduced by adding surface terms to a freeparticle Lagrangian in [28].

Motivated by the above mentioned results in differential geometry, we discuss in this paper the hidden symmetries corresponding to the fractional Killing vectors and KillingYano tensors on curved spaces deeply related to physical systems. 
The Caputo partial differential operator of fractional order $\alpha$ is defined as

$$
\begin{aligned}
& { }_{a} \partial_{x}^{\alpha} f(x, y) \\
& \equiv \begin{cases}\frac{1}{\Gamma(n-\alpha)} & \\
\times \int_{a}^{x}(x-u)^{n-\alpha-1} \frac{\partial^{n} f(u, y)}{\partial u^{n}} d u, & n-1<\alpha<n \\
\frac{\partial^{n}}{\partial x^{n}} f(x, y) . & \alpha=n\end{cases}
\end{aligned}
$$

Again in this work we consider the case $a=0, n-1<\alpha \leq n$, and we drop the term $a$ in the notation.

\section{The Main Results}

In the following, we present the Killing vectors and KillingYano tensors corresponding to some curved spaces with some physical significance.

2.1. One-Dimensional Case. Consider the one-dimensional free Lagrangian, admitting a constant of motion; that is, momentum [28]

$$
L=\frac{1}{2} \dot{x}^{2}+\dot{\lambda}_{2} \dot{x}
$$

The Lagrangian can be rewritten as

$$
L=\frac{1}{2} g_{i j} \dot{u}^{i} \dot{u}^{j},
$$

where $g_{i j}=\left[\begin{array}{ll}1 & 1 \\ 1 & 0\end{array}\right]$. The fractional Lagrangian of order $q$ is given by

$$
L=\frac{1}{2} g_{i j} D_{t}^{q} u^{i} D_{t}^{q} u^{j}
$$

where we consider the Caputo fractional derivative.

We generalize the Christoffel symbols in the fractional case, of order $n-1<q<n$, as

$$
{ }^{q} \Gamma_{\beta \mu}^{\gamma}=\frac{1}{2} g^{\alpha \gamma}\left(\partial_{\mu}^{q} g_{\alpha \beta}+\partial_{\beta}^{q} g_{\alpha \mu}-\partial_{\gamma}^{q} g_{\beta \mu}\right),
$$

where the partial derivatives of order $q$ are defined in the fractional case.

We notice that because the metric is constant, all the Christoffel symbols vanish,

$$
{ }^{q} \Gamma_{\mu \nu}^{\gamma}=0 .
$$

2.1.1. Fractional Killing Vectors and Killing-Yano Tensors. The Killing vectors can be calculated from the generalized equations, namely,

$$
V_{\alpha ; \beta}^{q}+V_{\beta ; \alpha}^{q}=0
$$

where $V_{\alpha ; \beta}^{q}$ is the fractional covariant derivative defined as

$$
V_{\alpha ; \beta}^{q}=\partial_{\beta}^{q} V_{\alpha}+g_{\alpha \mu}{ }^{q} \Gamma_{\delta \beta}^{\mu} g^{\delta \lambda} V_{\lambda}
$$

Because all the Christoffel symbols vanish, it is easy to show that

$$
\begin{aligned}
V_{1 ; 1}^{q} & =\partial_{1}^{q} V_{1}=0, \\
V_{2 ; 2}^{q} & =\partial_{2}^{q} V_{2}=0, \\
V_{1 ; 2}^{q}+V_{2 ; 1}^{q} & =V_{1,2}^{q}+V_{2,1}^{q}=\partial_{2}^{q} V_{1}+\partial_{1}^{q} V_{2}=0,
\end{aligned}
$$

For $0<q \leq 1$, a solution of the above equations is $V_{1}=-c y^{q}$, $V_{2}=c x^{q}$, where $c$ is a constant. While for $q>1$, we have the general solution

$$
\begin{aligned}
& V_{1}=-c y^{q}+\sum_{k=0}^{n-1}\left(a_{k} x^{k}+b_{k} y^{k}\right), \\
& V_{2}=c x^{q}+\sum_{k=0}^{n-1}\left(a_{k}^{\prime} x^{k}+b_{k}^{\prime} y^{k}\right),
\end{aligned}
$$

where $c, a_{k}, b_{k}, a_{k}^{\prime}, b_{k}^{\prime}$ are constants.

The fractional Killing-Yano antisymmetric tensor ${ }^{q} f_{\mu \nu}$ can be calculated using the condition

$$
{ }^{q} f_{\mu v ; \lambda}+{ }^{q} f_{\lambda v ; \mu}=0
$$

where ${ }^{q} f_{\mu \nu ; \lambda}$ is the fractional covariant derivative of the Killing-Yano tensor ${ }^{q} f_{\mu \nu}$ defined as

$$
{ }^{q} f_{\mu \nu ; \lambda}=\partial_{\lambda}^{q} f_{\mu \nu}-f_{\alpha \nu}{ }^{q} \Gamma_{\lambda \mu}^{\alpha}-f_{\mu \alpha}{ }^{q} \Gamma_{\lambda \nu}^{\alpha} .
$$

We find that

$$
\partial_{\lambda}^{q} f_{\mu \nu}=0
$$

for all values of $\lambda, \nu, \mu$. A solution is $f_{11}=f_{22}=0$ and $f_{12}=$ $c=-f_{21}$, where $c$ is a constant and for $0<q \leq 1$. While for $q>1$, that is, $n \geq 2$, we have the general solution

$$
f_{12}=-f_{21}=\sum_{k=0}^{n-1}\left(a_{k} x^{k}+b_{k} y^{k}\right),
$$

where $a_{k}, b_{k}$ are constants.

2.2. Two-Dimensional Case. Below we consider the classical free Lagrangian, in two dimensions, admitting a constant of motion; that is, angular momentum [28]

$$
L=\frac{1}{2}\left(\dot{x}^{2}+\dot{y}^{2}\right)+\dot{\lambda}_{3}(x \dot{y}-y \dot{x}) .
$$

The fractional Lagrangian is given by

$$
L=\frac{1}{2} g_{i j} D^{\alpha} q^{i} D^{\alpha} q^{j}
$$

where $g_{i j}$ is given by

$$
g_{i j}=\left[\begin{array}{ccc}
1 & 0 & -y \\
0 & 1 & x \\
-y & x & 0
\end{array}\right]
$$


The inverse matrix of the metric is

$$
g^{i j}=\frac{1}{x^{2}+y^{2}}\left[\begin{array}{ccc}
x^{2} & x y & -y \\
x y & y^{2} & x \\
-y & x & -1
\end{array}\right] .
$$

We generalize the Christoffel symbols in the fractional case, of order $n-1<q<n$, as

$$
{ }^{q} \Gamma_{\beta \mu}^{\gamma}=\frac{1}{2} g^{\alpha \gamma}\left(\partial_{\mu}^{q} g_{\alpha \beta}+\partial_{\beta}^{q} g_{\alpha \mu}-\partial_{\gamma}^{q} g_{\beta \mu}\right) \text {. }
$$

One can show that

$$
{ }^{q} \Gamma_{\mu \mu}^{\gamma}=0
$$

for $\gamma, \mu=1,2,3$, while

$$
\begin{aligned}
& { }^{q} \Gamma_{12}^{\gamma}=\frac{g^{3 \gamma}}{2}\left(\partial_{1}^{q} g_{32}+\partial_{2}^{q} g_{31}\right), \\
& { }^{q} \Gamma_{13}^{\gamma}=\frac{g^{2 \gamma}}{2}\left(\partial_{1}^{q} g_{32}-\partial_{2}^{q} g_{31}\right), \\
& { }^{q} \Gamma_{23}^{\gamma}=\frac{g^{1 \gamma}}{2}\left(\partial_{2}^{q} g_{13}+\partial_{1}^{q} g_{23}\right) .
\end{aligned}
$$

2.2.1. Fractional Killing Vectors. The Killing vectors can be calculated from the generalized equations

$$
V_{\alpha ; \beta}^{q}+V_{\beta ; \alpha}^{q}=0
$$

where $V_{\alpha ; \beta}^{q}$ is the fractional covariant derivative defined as

$$
V_{\alpha ; \beta}^{q}=\partial_{\beta}^{q} V_{\alpha}+g_{\alpha \mu}{ }^{q} \Gamma_{\delta \beta}^{\mu} g^{\delta \lambda} V_{\lambda}
$$

It is easy to show that

$$
\begin{gathered}
V_{1 ; 1}^{q}=\partial_{1}^{q} V_{1}=0, \\
V_{2 ; 2}^{q}=\partial_{2}^{q} V_{2}=0, \\
V_{3 ; 3}^{q}=\partial_{3}^{q} V_{3}=0, \\
V_{1 ; 2}^{q}+V_{2 ; 1}^{q}=V_{1,2}^{q}+V_{2,1}^{q}=\partial_{2}^{q} V_{1}+\partial_{1}^{q} V_{2}=0, \\
V_{1 ; 3}^{q}+V_{3 ; 1}^{q}=\partial_{3}^{q} V_{1}+\partial_{1}^{q} V_{3}+g^{2 \lambda} V_{\lambda} \partial_{2}^{q} g_{13}=0, \\
V_{2 ; 3}^{q}+V_{3 ; 2}^{q}=\partial_{3}^{q} V_{2}+\partial_{2}^{q} V_{3}+g^{1 \lambda} V_{\lambda} \partial_{1}^{q} g_{23}=0 .
\end{gathered}
$$

A solution for $V_{1}$ and $V_{2}$ can be easily found for any fractional order $q$, that is, $n-1<q<n$, namely,

$$
\begin{gathered}
V_{1}=c y^{q}+\sum_{k=0}^{n-1}\left(a_{k} x^{k}+b_{k} y^{k}\right), \\
V_{2}=-c x^{q}+\sum_{k=0}^{n-1}\left(c_{k} x^{k}+d_{k} y^{k}\right),
\end{gathered}
$$

where $c, a_{k}, b_{k}, c_{k}, d_{k}$ are constants. The solution to $V_{3}$ is not easy to find for $0<q \leq 1$. However, for $n \geq 2$, that is, $1<q$, the equations simplify because

$$
\partial_{2}^{q} g_{13}=\partial_{1}^{q} g_{23}=0
$$

In this case a general solution is obtained as

$$
V_{3}=\sum_{k=0}^{n-1}\left(a_{k}^{\prime} x^{k}+b_{k}^{\prime} y^{k}\right),
$$

where $a_{k}^{\prime}, b_{k}^{\prime}$ are constants.
2.2.2. Fractional Killing-Yano Tensors. The fractional antisymmetric Killing-Yano tensors can be derived using the condition that

$$
{ }^{q} f_{\mu v ; \lambda}+{ }^{q} f_{\lambda v ; \mu}=0
$$

where ${ }^{q} f_{\mu \nu ; \lambda}$ is the fractional covariant derivative of the Killing-Yano tensor ${ }^{q} f_{\mu \nu}$ defined as

$$
{ }^{q} f_{\mu \nu ; \lambda}=\partial_{\lambda}^{q} f_{\mu \nu}-f_{\alpha \nu}{ }^{q} \Gamma_{\lambda \mu}^{\alpha}-f_{\mu \alpha}{ }^{q} \Gamma_{\lambda \nu}^{\alpha} .
$$

For the fractional order $0<q<1$, it is difficult to find an analytic solution. However, for the order $q>1$, the Christoffel symbols vanish; we find that

$$
\partial_{\lambda}^{q} f_{\mu \nu}=0
$$

for all values of $\lambda, \nu, \mu$. A solution is that $f_{11}=f_{22}=f_{33}=0$ and $f_{12}, f_{13}, f_{23}$ are a linear combination of $x^{k}, y^{k}$ where $k=$ $0,1,2, \ldots, n-1$, namely,

$$
\begin{aligned}
& f_{12}=-f_{21}=\sum_{k=0}^{n-1}\left(a_{k} x^{k}+b_{k} y^{k}\right), \\
& f_{13}=-f_{31}=\sum_{k=0}^{n-1}\left(a_{k}^{\prime} x^{k}+b_{k}^{\prime} y^{k}\right), \\
& f_{23}=-f_{32}=\sum_{k=0}^{n-1}\left(c_{k} x^{k}+d_{k} y^{k}\right),
\end{aligned}
$$

where $a_{k}, b_{k}, a_{k}^{\prime}, b_{k}^{\prime}, c_{k}, d_{k}$ are constants.

\section{Conclusion}

In this work, we investigate the existence of fractional Killing vectors and Killing-Yano tensors for the geometry induced by fractionalizing the classical free Lagrangian admitting a constant of motion. We discuss the cases of one-dimensional and two-dimensional curved space. We use the Caputo definition of the fractional derivative to calculate the fractional Christoffel symbols and consequently we provide explicit solution to the fractional Killing vectors and Killing-Yano tensors.

\section{Conflict of Interests}

The authors declare that there is no conflict of interests regar ding the publication of this paper.

\section{References}

[1] A. A. Kilbas, H. M. Srivastava, and J. J. Trujillo, Theory and Applications of Fractional Diffdrential Equations, vol. 204, Elservier, Amsterdam, The Netherlands, 2006.

[2] V. Lakshmikantham and A. S. Vatsala, "Basic theory of fractional differential equations," Nonlinear Analysis: Theory, Methods \& Applications, vol. 69, pp. 2677-2682, 2009.

[3] I. Podlubny, Fractional Differential Equations, Academic Press, San Diego, Calif, USA, 1999. 
[4] J. Sabatier, O. P. Agrawal, and J. A. T. Machado, Advances in Fractional Calculus: Theoretical Developments and Applications in Physics and Engineering, Springer, Dordrecht, The Netherlands, 2007.

[5] K. S. Miller and B. Ross, An Introduction to the Fractional Calculus and Fractional Differential Equations, John Wiley \& Sons, 1993.

[6] K. B. Oldham and J. Spanier, The Fractional Calculus; Theory and Applications of Differentiation and Integration to Arbitrary Order(Mathematics in Science and Engineering, V), Academic Press, 1974.

[7] R. Herrmann, Fractional Calculus. An Introduction for Physicists, World Scientific, Singapore, 2011.

[8] S. G. Samko, A. A. Kilbas, and O. I. Marichev, Fractional Integral and Derivatives, Theory and Applications, Gordon and Breach, Yverdon, Switzerland, 1993.

[9] D. Baleanu, K. Diethelm, E. Scalas, and J. J. Trujillo, Fractional Calculus Models and Numerical Methods, World Scientific, Singapore, 2012.

[10] F. Mainardi, "Fractional calculus: some basic problems in continuum and statistical mechanics," in Fractals and Fractional Calculus in Continuum Mechanics, pp. 291-348, Springer, Vienna, Austria, 1997.

[11] R. Hilfer, Application of Fractional Calculus in Physics, World Scientific Publishing Company, Singapore, 2000.

[12] O. P. Agrawal, "Formulation of Euler-Lagrange equations for fractional variational problems," Journal of Mathematical Analysis and Applications, vol. 272, no. 1, pp. 368-379, 2002.

[13] X.-J. Yang, Advanced Local Fractional Calculus and Its Applications, World Science, New York, NY, USA, 2012.

[14] Y. Zhao, D. Baleanu, C. Cattani, D. Cheng, and X.-J. Yang, "Maxwell's equations on cantor Sets: a local fractional approach," Advances in High Energy Physics, vol. 2013, Article ID 686371, 6 pages, 2013.

[15] D. Baleanu and S. I. Vacaru, "Fedosov quantization of fractional lagrange spaces," International Journal of Theoretical Physics, vol. 50, no. 1, pp. 233-243, 2011.

[16] D. Baleanu and S. I. Vacaru, "Fractional almost Kähler-Lagrange geometry," Nonlinear Dynamics, vol. 64, no. 4, pp. 365-373, 2011.

[17] M. Visinescu, "Geodesic motion in Taub-NUT spinning space," Classical and Quantum Gravity, vol. 11, no. 7, pp. 18671879, 1994.

[18] G. W. Gibbons, R. H. Rietdijk, and J. W. Van Holten, "SUSY in the sky," Nuclear Physics B, vol. 404, no. 1-2, pp. 42-64, 1993.

[19] J. W. van Holten, "Supersymmetry and the geometry of TaubNUT," Physics Letters, Section B: Nuclear, Elementary Particle and High-Energy Physics, vol. 342, no. 1-4, pp. 47-52, 1995.

[20] D. Baleanu and Ö. Defterli, "Killing-Yano tensors and angular momentum," Czechoslovak Journal of Physics, vol. 54, no. 2, pp. 157-165, 2004.

[21] D. Baleanu and S. Başkal, "Dual metrics and nongeneric supersymmetries for a class of siklos space-times," International Journal of Modern Physics A, vol. 17, no. 26, pp. 3737-3747, 2002.

[22] D. Garfinkle and E. N. Glass, "Killing tensors and symmetries," Classical and Quantum Gravity, vol. 27, no. 9, Article ID 095004, 2010.

[23] Sh. Tachibana, "On conformal Killing tensor in a Riemannian space," Tohoku Mathematical Journal, vol. 21, no. 2, pp. 56-64, 1969.
[24] T. Houri and K. Yamamoto, "Killing-Yano symmetry of KaluzaKlein black holes in five dimensions," Classical and Quantum Gravity, vol. 30, no. 7, Article ID 075013, 21 pages, 2013.

[25] D. Baleanu and A. Karasu, "Lax tensors, killing tensors and geometric duality," Modern Physics Letters A, vol. 14, no. 37, pp. 2587-2594, 1999.

[26] D. Baleanu and S. Bakal, "Geometrization of the lax pair tensors," Modern Physics Letters A, vol. 15, no. 24, pp. 1503-1510, 2000.

[27] K. Cottrill-Shepherd and M. Naber, "Fractional differential forms," Journal of Mathematical Physics, vol. 42, no. 5, pp. 22032212, 2001.

[28] Y. Güler, D. Baleanu, and M. Cenk, "Surface terms, angular momentum and Hamilton-Jacobi formalism," Nuovo Cimento della Societa Italiana di Fisica B, vol. 118, no. 3, pp. 293-306, 2003. 


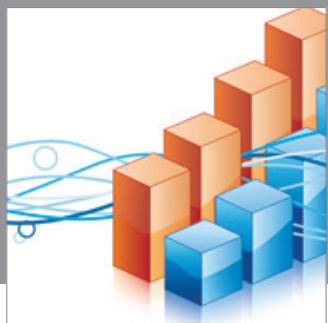

Advances in

Operations Research

mansans

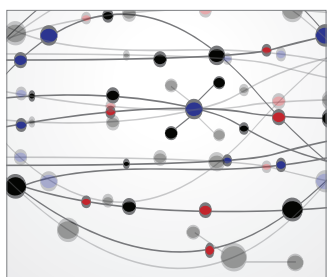

The Scientific World Journal
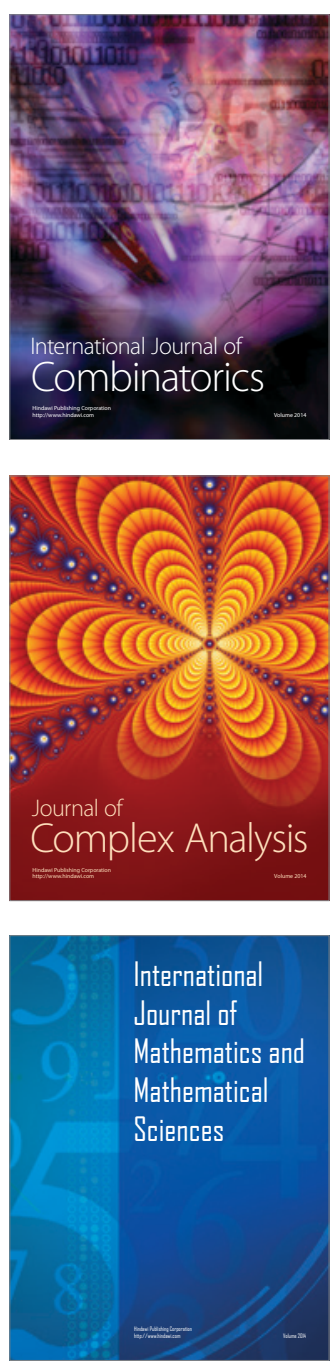
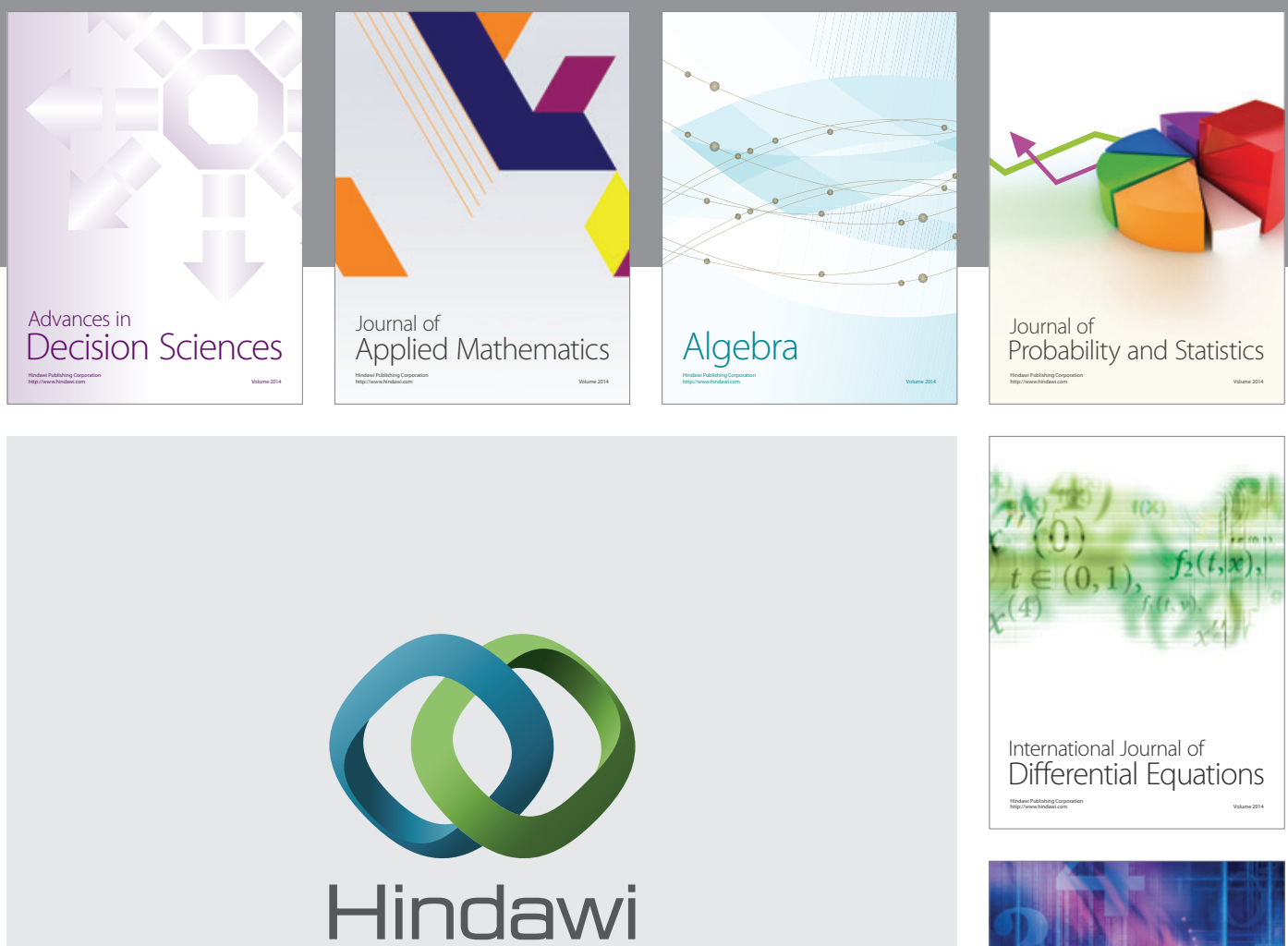

Submit your manuscripts at http://www.hindawi.com
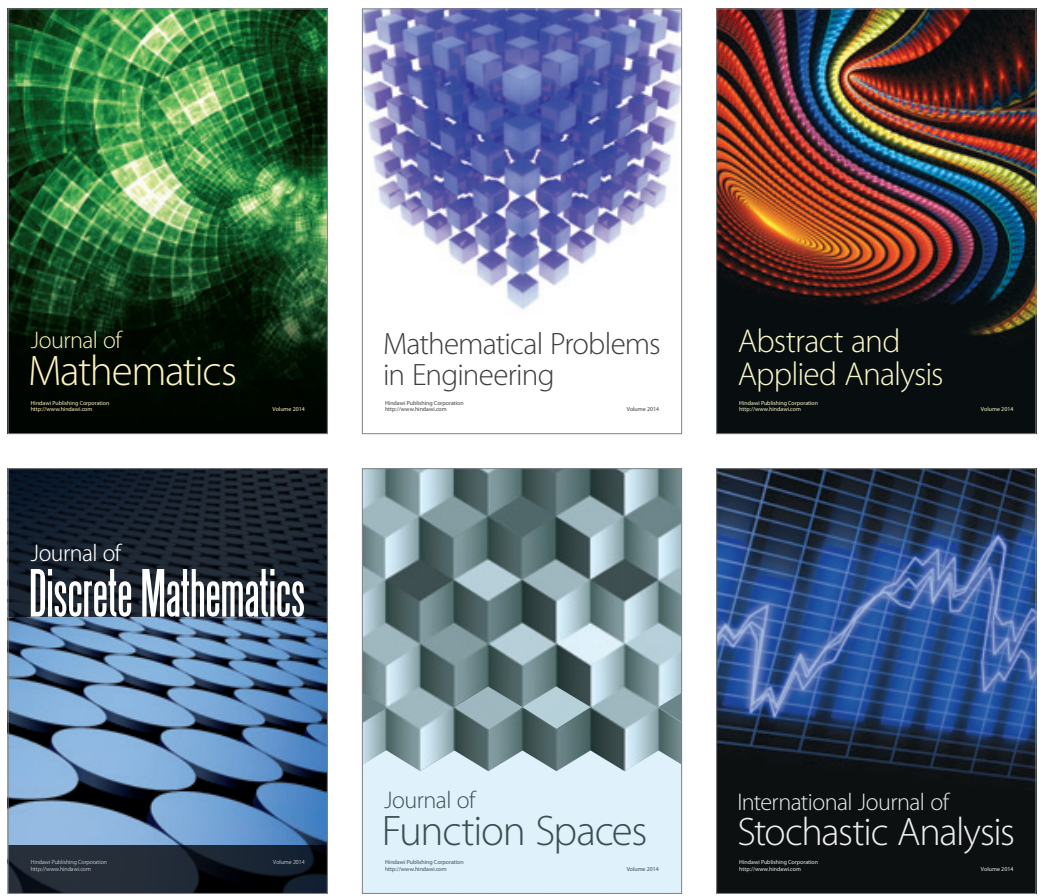

Journal of

Function Spaces

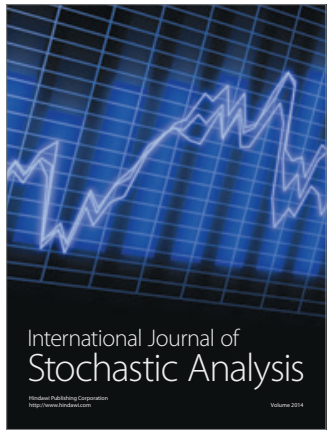

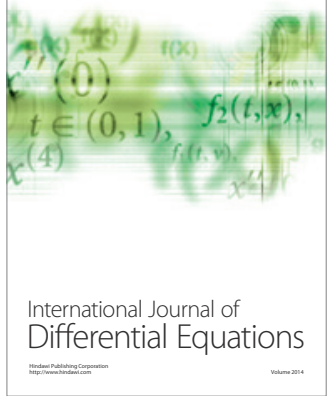
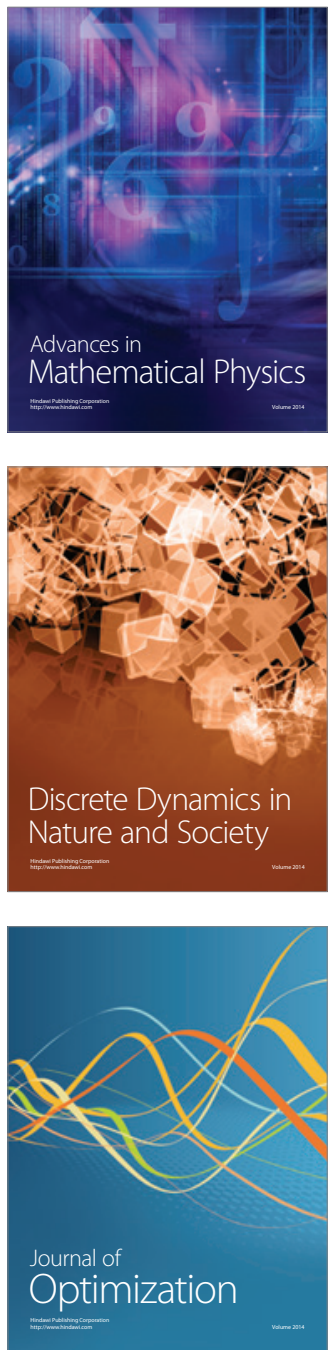